\title{
TOTAL CO-INDEPENDENT DOMINATION OF JUMP GRAPH
}

\author{
M. Karthikeyan ${ }^{1 \S}$, A. Elumalai ${ }^{2}$ \\ ${ }^{1}$ Bharathiar University \\ Coimbatore, 641046, INDIA \\ ${ }^{1}$ Department of Mathematics \\ Panimalar Engineering College \\ Chennai, 600123, INDIA \\ ${ }^{2}$ Department of Mathematics \\ Valliammai Engineering College \\ Chennai, 603203, INDIA
}

\begin{abstract}
A set $D \subseteq V[J(G)]$ is dominating set of jump graph, if every vertex not in $D$ is adjacent to vertex in $D$. The domination number of the jump graph is the minimum cardinality of dominating set of jump graph $J(G)$. A total dominating set $S$ of a graph $G$ is called Co-independent dominating set if the induced subgraph $\langle G\rangle$ has no edge and has at least one vertex. In this paper, we introduce the total Co-independent domination of jump graph, and its exact values for some standard graphs and some results are established.
\end{abstract}

AMS Subject Classification: $05 \mathrm{C} 78$

Key Words: dominating set, total dominating set, total co-independent dominating set, jump graph

\section{Introduction}

Let $G(p, q)$ be a graph with $p=|V|$ and $q=|E|$ denote the number of vertices and edges of a graph $G$, respectively. All the graphs considered here are finite, non-trivial, undirected and connected without loops or multiple edges.

Received:

Revised:

April 24, 2016

Published: September 20, 2016

October 21, 2016 (c) 2016 Academic Publications, Ltd. url: www.acadpubl.eu

${ }^{\S}$ Correspondence author 
In general, we use $\langle X\rangle$ to denote the subgraph induced by the set of vertices $X$. A vertex of degree one is called a pendent vertex. A vertex adjacent to pendent vertex is called the support vertex. The maximum $d(u, v)$ for all $u$ in $G$ is eccentricity of $v$ and the maximum eccentricity is the diameter $\operatorname{diam}(G)$. The circumference of a graph $G$ with at least one cycle is the length of a longest cycle in $G$ and it is denoted by $\operatorname{circum}(G)$. As usual $P_{n}, C_{n}$ and $K_{n}$ are respectively, the path, cycle and complete graph of order $n, K_{r, s}$ is the complete bipartite graph with two partite sets containing $r$ and $s$ vertices. A set $D$ of vertices in a graph $G$ is a dominating set if every vertex in $V-D$ is adjacent to some vertex in $D$. The domination number $\gamma(G)$ is the minimum cardinality of a dominating set of $G$. For terminology and notations not specifically defined here we refer reader to [1][2].For more details about domination number and its related parameters, we refer to [3][4][5].

A dominating set $S$ of $G$ is called a connect dominating set if the induced subgraph $\langle S\rangle$ is connected. The minimum cardinality of a connected dominating set of $G$ is called the connected domination number of $G$ and is denoted by $\gamma_{c}(G)[6]$.A dominating set $S$ of $G$ is called total dominating set if the induced subgraph $\langle S\rangle$ has no isolated vertices. The minimum cardinality of a total dominating set of $G$ is called the total domination number of $G$ and is denoted by $\gamma_{t}(G)[3]$.

Definition 1. The line graph $L(G)$ of $G$ has the edges of $G$ as its vertices which are adjacent in $L(G)$ if and only if the corresponding edges are adjacent in $G$. We call the complement of line graph $L(G)$ as the jump graph $J(G)$ of $G$ ,found in [7]. The jump graph $J(G)$ of a graph $G$ is the graph defined on $E(G)$ and in which two vertices are adjacent if and only if they are not adjacent in $G$. Since both $L(G)$ and $J(G)$ are defined on the edge set of a graph $G$.

Remark 2. The isolated vertices of $G$ (if $G$ has) play no role in line graph and jump graph transformation. Here we assume that the graph $G$ under consideration is non-empty and has no isolated vertices found in [7].

$\{2,3\}$ is the minimum total dominating set. Hence $\gamma_{t}(G)=2$, clearly $\{2,3\}$ is not total Co-independent dominating set of $\mathrm{G}$. The minimum total Coindependent dominating sets are $\{1,2,3,4\},\{1,2,3,5\},\{2,3,4,9\},\{2,3,5,9\}$. Therefore $\gamma_{t, \text { coi }}(G)=4$.

As in the standard dominating set, any minimum total Co-independent dominating set is minimal, but the converse is not true as seen in figure 1. The set $\{1,2,4,5,6,7\}$ is minimal total Co-independent dominating set but not minimum total Co-independent dominating set.

Definition 3. A total dominating set $S$ of a $J(G)$ is called total Co- 


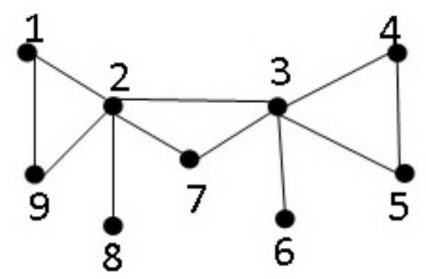

Figure 1

independent dominating set if the induced subgraph $\langle V-S\rangle$ has no edge and has at least one vertex. The minimum cardinality of a total Co-independent dominating set of $J(G)$ is called the total Co-independent domination number of $J(G)$ and is denoted by $\gamma_{t, c o i} J(G)$.

\section{Main Results}

Observation. For any Cycle $C_{p}$, with $p \geq 5, \gamma_{t, c o i}\left[J\left(C_{p}\right)\right]=4$.

Observation. For any path $P_{p}$, with $p \geq 5, \gamma_{t, c o i}\left[J\left(P_{p}\right)\right]=4$.

Observation. For any complete graph $K_{p}$, with $p \geq 5, \gamma_{t, c o i}\left[J\left(K_{p}\right)\right]=6$.

Observation. For any connected $(p, q)$ graph $G, \gamma_{t, c o i}[J(G)]=q$.

Observation. For any complete bipartite graph $K_{m, n}$

$$
\gamma_{t, \text { coi }}\left[J\left(K_{m, n}\right)\right]=\left\{\begin{array}{l}
4 \text { for } k_{2, n} \text { where } n \geq 2 \\
6 \text { for } k_{m, n} \text { where } m, n \geq 3
\end{array}\right.
$$

Observation. For any wheel $W_{p}$,

$$
\gamma_{t, c o i}\left[J\left(W_{p}\right)\right]=\left\{\begin{array}{l}
6 \text { for } p=5,6 \\
4 \text { for } p \geq 7
\end{array}\right.
$$

Theorem 4. For any connected graph $G$ With diameter, $\operatorname{diam}(G) \geq 2$, $\gamma_{t, \text { coi }} J(G) \geq 4$.

Proof. Let uv be a path of maximum distance in $\mathrm{G}$. Then $\mathrm{d}(\mathrm{u}, \mathrm{v})=\operatorname{diam}(\mathrm{G})$ We can prove the theorem with the following cases.

Case (i): For $\operatorname{diam}(G)=2$, choose a vertex $V_{1}$ of eccentricity 2 with maximum degree among others. Let $V_{1}=\left\{v_{1}, v_{2}, \cdots\right\}$ corresponding to the elements of $\left\{e_{1}, e_{2}, \cdots\right\}$ forming a total Co-independent dominating set in jump 
graph $\mathrm{J}(\mathrm{G})$.Every vertex $u \notin V_{1}$ is adjacent to a vertex in $V_{1}$. Hence $V_{1}$ is a minimum total Co-independent dominating set. So the total Co-independent domination number of the jump graph will be equal to the degree of $V_{1}$ we say $\gamma_{t, \text { coi }}[J(G)]>4$.

Case (ii): For $\operatorname{diam}(G)>2$.Let $e_{1}$ be any edge adjacent to $\mathrm{u}$ and $e_{2}$ be any edge adjacent to v. Let $\left\{e_{1}, e_{2}\right\} \subseteq E(G)$ form a corresponding vertex set $v_{1}, v_{2} \subseteq V(J(G))$. These two vertices form total Co-independent dominating set in jump graph. Since these vertices $v_{1}, v_{2}$ are adjacent to all other vertices of $V\left(J(G)\right.$ ), it follows that $v_{1}, v_{2}$ becomes a minimum total Co-independent dominating set. Hence $\gamma_{t, c o i}[J(G)]=4$. In view of above cases, we can conclude that for any connected graph $G, \gamma_{t, c o i}[J(G)] \geq 4$.

Theorem 5. For any connected graph $G$ with circumference $\operatorname{circum}(G) \geq$ $4, \gamma_{t, \text { coi }}[J(G)] \geq 4$.

Proof. Let $u-v-u$ be a longest cycle in $G$. Then $C(G)=\operatorname{circum}(G)$. We can prove the theorem with the following cases.

Case i: For $\operatorname{Circum}(G)=4$, choose a vertex $V_{1}$ that have longest cycle among others. Let $V_{1}=\left\{v_{1}, v_{2}, \ldots\right\}$ corresponding to the elements of $\left\{e_{1}, e_{2}, \ldots\right\}$ forming a total Co-independent dominating set in jump graph $J(G)$.Every vertex $u \notin V_{1}$ is adjacent to a vertex in $V_{1}$. Hence $V_{1}$ is a minimum total Coindependent dominating set. So the total Co-independent domination number of the jump graph will be equal to the longest cycle. We say $\gamma_{t, c o i}[J(G)]>4$.

Case ii: For $\operatorname{Circum}(G)>4$. Let $e_{1}$ be any edge adjacent to $u$ and $e_{2}$ be any edge adjacent to $v$. Let $\left\{e_{1}, e_{2}\right\} \subseteq E(G)$ form a corresponding vertex set $\left\{v_{1}, v_{2}\right\} \subseteq V(J(G))$. These two vertices form total Co-independent dominating set in jump graph. Since these vertices $\left\{v_{1}, v_{2}\right\}$ are adjacent to all other vertices of $V(J(G))$, it follows that $\left\{v_{1}, v_{2}\right\}$ becomes a minimum total Co-independent dominating set. Hence $\gamma_{t, c o i}[J(G)]=4$. In view of above cases, we can conclude that for any connected graph $G, \gamma_{t, c o i}[J(G)] \geq 4$.

Theorem 6. For any tree $T$ with diameter greater than $3, \gamma_{t, c o i}[J(T)]=q$.

Proof. If the diameter is less than or equal to 3, then the jump graph will be disconnected. Let $u v$ be a path of maximum length in a tree $T$ where diameter is greater than 3 . Let $e_{i}$ be the pendant edge adjacent to $u$ and $e_{k}$ be the pendant edge adjacent to $v$. The vertex set $\left\{v_{i}, v_{k}\right\}$ of $J(T)$ corresponding to the edges of $\left\{e_{i}, e_{k}\right\}$ in $T$ will form total Co-independent dominating set in 
$J(T)$. Since all the other vertices of $V[J(T)]$ are adjacent with $\left\{v_{i}, v_{k}\right\}$, it forms a minimum total Co-independent dominating set. Hence $\gamma_{t, c o i}[J(T)]=q$.

Theorem 7. For any jump graph $J(G)=(V, E)$ with no isolated vertices and $|V| \geq 3, \gamma(J(G)) \leq \gamma_{t}(J(G)) \leq \gamma_{t, c o i}(J(G))$.

Proof. Let $J(G)=(V, E)$ be a graph with no isolated vertices. Suppose that $S \subseteq V$ is any minimum total Co-independent dominating set of $J(G)$. Since for any jump graph $J(G)$ any total Co-independent dominating set $S$ is also total dominating set and every total dominating set is also dominating set. Hence $\gamma(J(G)) \leq \gamma_{t}(J(G)) \leq \gamma_{t, c o i}(J(G))$.

Theorem 8. let $J(G)$ be a jump graph with $D$ as minimal total Coindependent dominating set, then $V-D$ is independent dominating set of $J(G)$.

Proof. Let $D$ be minimal total Co-independent dominating set of $J(G)$. Suppose that $V-D$ is not independent dominating set of $J(G)$, since $D$ is total Co-independent dominating set of $G$ then $V-D$ is independent set, that means if we suppose that $V-D$ is not independent dominating set of $J(G)$, then there exists a vertex $u$ such that $u$ is not dominated by any vertex in $V-D$. Since $J(G)$ has total Co-independent dominating set, then $J(G)$ has no isolated vertices, therefore $u$ is dominated by at least one vertex in $D-\{u\}$. Thus $D-\{u\}$ is total Co-independent dominating set of $J(G)$, which contradicts the minimality of $D$. Thus every vertex in $D$ is adjacent with at least one vertex in $V-D$ and $V-D$ is independent set. Hence $V-D$ is independent dominating set of $J(G)$.

Theorem 9. For any $J(G)$ any total Co-independent dominating set of $J(G)$ contains all the support vertices.

Proof. Suppose the graph $J(G)$ has a total Co-independent dominating set $D$ and let $V$ be support vertex does not belongs to $D$ then clearly the pendent vertex which adjacent to $V$ cannot belong to $D$ from the definition of total Co-independent dominating set. Hence $D$ is not a dominating set, which is contradiction. 


\section{Conclusion}

Thus we conclude the total Co-independent domination number and the parameters of total Co-independent domination number of jump graphs like $k_{p}$, $C_{p}, P_{p}, W_{p}$ and $K_{m, n}$.

\section{References}

[1] F. Harary, Graph Theory, Addision-Wesley, Reading Mass, 1969.

[2] J. Bondy and U. Murthy, Graph Theory with Applications, North Holland. New York, 1976.

[3] T.H. Haynes, S.T. Hedetniemi and P.J. Slater, Fundamentals of Domination in Graphs, Marcel Dekker, Inc, New York, 1998.

[4] E. Sampathkumar and P.S. Neeralagi, The neighborhood number of a graph, Indian. J. Pure and Appl. Math., 16, No. 2 (1985) 126-132.

[5] H.B. Walikar, B.D. Acharya and E. Sampathkumar, Recent Developments in Theory of Domination in Graphs, Mehta Research institute Alahabad, MRI lecture Notes in Math., 1, 1979.

[6] E. Sampathkumar and H.B.Walikar, The connected domination number of a graph, J. Math Phys. Sci., 13 (1979), 607-613.

[7] G. Chartrand, H. Hevia, E.B. Jarrett, M. Schultz, Subgraph distances in graphs defined by edge transfers, Discrete Math., 170 (1997), 63-79.

[8] N.D. Soner, B.V. Dhananjayamurthy, G. Deepak, Total co-independent domination in graphs, Appl. Math. Sci., 6, No. 131 (2012), 6545-6551. 